The CO bar has a double-peaked structure with a shallow dip toward the $2.2 \mu \mathrm{m}$ nucleus, being symmetric with respect to it. The two maxima lie about $200 \mathrm{pc}$ away from the nucleus. The molecular hydrogen mass of the nuclear bar is estimated to be $\sim 2 \times 10^{8} \mathrm{M}_{\odot}$; five percent of the total $\mathrm{H}_{2}$ mass is accumulated in the central small area of the galaxy. This may explain the vigorous star forming activity in the nucleus of IC 342 .

The beam deconvolved width (FWHM) of the bar is $0.6 \mathrm{kpc}$ and is considerably wider than that measured by Lo et al. (1984). The narrower width of their $\mathrm{CO}$ map might be caused by the limited velocity coverage of their spectrometer.

\title{
REFERENCES
}

Lo, K.Y. et al.: 1984, Astrophys. J. Letters 282, L59.

Becklin, E.E., Gatley, I., Matthews, K., Neugebauder, G., Sellgren, K., Werner, M.W., and Wynn-Williams, C.G.: 1980, Astrophys. J. 236, 441 .

\section{MOLECULAR CLOUDS IN BARRED GALAXIES}

F. Combes, M. Gérin

Radioastronomy Observatoire de Meudon, France

A stellar bar is an essential dynamical component for the behaviour of gas and molecular clouds in a spiral galaxy. It can trigger the formation of the arms and provokes radial transfer of gas. N-body simulations have shown that the bar forms easily and is very stable; according to classification, the majority of spiral galaxies are barred.

We have simulated the molecular clouds response to a bar forcing in a spiral galaxy, taking into account: collisions between clouds and the consequent dissipation of energy, the formation of giant molecular clouds by coalescence, the heating of the interstellar medium by star formation and the subsequent dissipation of giant clouds in small entities (cf. Combes and Gerin 1985). Star formation is enhanced in spiral arms because of the increased collision rate between clouds and the induced formation of GMC. This is due to the particular alignment of orbits in a bar potential: orbits crowding in spiral arms increases the density and the relative velocity of clouds, both favoring collisions. However, the equilibrium is never reached in the molecular clouds medium, since the arm crossing time is shorter than the collisional equilibrium time. In the arms, the GMC formation time becomes comparable to their lifetime, but it is much longer in the interarms. Because of the deficiency of GMC in the interarms, heating of the medium occurs essential$1 y$ at the exit of spiral arms. 
The gaseous final configuration depends essentially on the angular velocity of the bar and on the number of resonances in the disk. Nuclear rings form at the inner Lindblad resonances when the bar velocity is low. In this case, the molecular component is highly concentrated and star formation is enhanced in the inner parts of the galaxy. This could explain the rings of hot spots seen in many barred galaxies. Recent observations of galaxies in $\mathrm{CO}$ with high resolution reveal a barred distribution consistent with these results (cf. IC 342 Lo et al. 1984 , Hayashi et al. 1987, M83 Handa et al. 1987, NGC 6946 Ball et al. 1985, M82 Nakai et al. 1987, Lo et al. 1987).

\section{REFERENCES}

Bal1, R., Sargent, A.I., Scoville, N.Z., Lo, K.Y., Scott, S.L.: 1985, Astrophys. J. 298, L21.

Combes, F., Gerin, M.: 1985, Astron. Astrophys. 150, 327.

Handa, T., Sofue, Y., Nakai, N., Fujimoto, M., Hayashi, M.: 1987, these proceedings.

Hayashi, M., Handa, T., Sofue, Y., Hasegawa, T., Nakai, N., Lord, S., Young, J.: 1987, these proceedings.

Lo, K.Y., et al. 1984, Astrophys. J. 282, L59.

Lo, K.Y., Cheung, K.W., Masson, C.R., Phillips, T.G., Scoville, N.Z., Woody, D.P.: 1987, these proceedings.

Nakai, N., Hayashi, M., Hasegawa, T., Sofue, Y., Handa, T., Sasaki, M.: 1987, these proceedings.

ANEMIC GALAXIES: EVIDENCE FOR A THRESHOLD IN STAR FORMATION PROCESSES

B. Guiderdoni

Institut d'Astrophysique de Paris

98 bis Bd. Arago, F-75014, Paris, France

From a sample of Virgo Cluster and "field" disk galaxies, it is shown that a critical value of the HI surface density discriminates between RDDO anemic and "healthy" spirals. Below this threshold, at least massive stars do not form any more and the galaxy gets the anemic appearance. The influence of the HI content on the global star formation rate is discussed in the context of present models (Elmegreen 1979, Seiden and Gerola 1979, Dopita 1985), as well as the fate of disks in cluster and "field" environments. The existence of this threshold is an issue for the problem caused by the short gas consumption time scales derived from the observations of spiral galaxies. 